\title{
Sentiment classification of customer reviews based on fuzzy logic
}

\begin{abstract}
Nowadays, e-commerce is growing fast, so product reviews have grown rapidly on the web. The large number of reviews makes it difficult for manufacturers or businesses to automatically classify them into different semantic orientations (positive, negative, and neutral). Most existing method utilize a list of opinion words for sentiment classification. whereas, this paper propose a fuzzy logic model to perform semantic classifications of customers review into the following sub-classes: very weak, weak, moderate, very strong and strong by combinations adjective, adverb and verb to increase holistic the accuracy of lexicon approach. Fuzzy logic, unlike statistical data mining techniques, not only allows using nonnumerical values also introduces the notion of linguistic variables. Using linguistic terms and variables will result in a more human oriented querying process.
\end{abstract}

Keyword: Fuzzy logic; Opinion mining; Sentiment classification 